\title{
Do Pelvic Fractures in Elderly Occur without Trauma or Skele- tal Disease?
}

\section{David Collin* and Jan H Göthlin}

Department of Radiology, Sahlgrenska University Hospital, Sweden

*Corresponding author: David Collin, M.D. Ph.D, Department of Radiology, Sahlgrenska University Hospital, S-431 80 Mölndal, Sweden, Tel: +4631-342-0000, E-mail: david.collin@vgregion.se

\begin{abstract}
Background: Pelvic fractures in elderly caused by trauma or by osteoporosis are not uncommon. There is no reference material on pelvic fractures in subjects without trauma to pelvis or hip, osteoporosis or systemic disease.

Purpose: To assess whether pelvic fractures occur in a normal population over age 50 examined with MRI.

Material and Methods: A study cohort of 418 patients examined by pelvic MRI for non-trauma reasons without osteoporosis or systemic disease was collected and independently reviewed by two musculoskeletal radiologists. There were 173 males with mean age of 67 years (range 50-92) and 245 females with mean age 66 (range 50-90). Overall mean age was 67 years (range 50-92). The patients were grouped according to age and gender. 170 patients were in the age interval 70-92.
\end{abstract}

Results: Regardless of age there were no fractures in any of the study objects.

Conclusion: Pelvic fractures do not occur in normal patients without trauma.

\section{Keywords}

Reference standards, Pelvic bones fractures, Spontaneous fractures, Bone, Magnetic resonance imaging

\section{Introduction}

Pelvic fractures in elderly caused by trauma [1] or by osteoporosis [2] are not uncommon. In a study of 316 patients over age 50 subjected to fall trauma to the hip and with clinical suspicion of hip fracture, 132 (42\%) patients had exclusively pelvic fracture at MRI [1]. Perusal of the literature has not revealed any material on pelvic fractures in patients over age 50 without trauma to pelvis or hip, osteoporosis, systemic or neoplastic disease at MRI. The aim of the study was to produce a reference material with the hypothesis that elderly without trauma, osteoporosis, systemic or neoplastic disease do not have pelvic fractures demonstrated at MRI.

\section{Material and Methods}

For the period 2007-2016 all 829 patients over age 50 examined with MRI of the pelvis were collected from the PACS and RIS of the [to be replaced after review]. Excluded were patients with hip and pelvic trauma, hip or pelvic implants, bone metastases, previous radiotherapy of the pelvis or systemic diseases with impairment of bone quality such as renal disease or bone marrow metabolic disease. No patient had been referred for investigation of osteoporosis.

The study cohort consists of 418 patients examined with MRI for non-trauma reasons. There were 173 males with mean age of 67 years (range 50-92) and 245 females with mean age 66 (range 50-90). Overall mean age was 67 years (range 50-92). The patients were grouped according to age and gender. All coronal STIR and T1-sequences were retrieved and independently reviewed by two specialists well experienced in musculoskeletal radiology and with

Table 1: 1.5T MRI-scanners with protocol.

Philips, Achieva, ST 5 mm (STIR TR 4121, TE 110; T1 TR 505, TE 18)

GE, Genesis Signa, ST 4-5 mm (STIR TR 4080, TE 56; T1 TR 600 , TE 14)

Siemens, Magnetom Harmony Expert, ST $4 \mathrm{~mm}$ (STIR TR 4000, TE 112; T1 TR 565, TE 15)

Siemens, Symphony, ST 5 mm (STIR TR 4500, TE 54; T1 TR 520, TE 20)

MRI scanners used to examine the pelvis of 418 consecutive patients for non-trauma-related reasons.

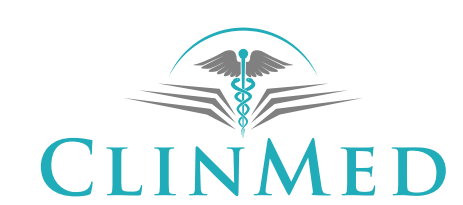

INTERNATIONAL LIBRARY

Citation: Collin D, Göthlin JH (2018) Do Pelvic Fractures in Elderly Occur without Trauma or Skeletal Disease?. Int J Radiol Imaging Technol 4:035. doi.org/10.23937/2572-3235.1510035

Accepted: June 04, 2018: Published: June 06, 2018

Copyright: (C) 2018 Collin D, et al. This is an open-access article distributed under the terms of the Creative Commons Attribution License, which permits unrestricted use, distribution, and reproduction in any medium, provided the original author and source are credited. 
Table 2: Stratification according to age in 418 patients.

\begin{tabular}{|l|l|l|l|l|}
\hline Years & Mean age & No of patients & Male/female (No) & Male/female ratio \\
\hline $50-59$ & 55 & 105 & $35 / 70$ & 0.5 \\
\hline $60-69$ & 64 & 143 & $62 / 81$ & 0.77 \\
\hline $70-79$ & 74 & 119 & $59 / 60$ & 0.98 \\
\hline $80-92$ & 84 & 51 & $17 / 34$ & 0.5 \\
\hline Total & 67 & 418 & $173 / 245$ & 0.71 \\
\hline
\end{tabular}

No fractures were found in any age group. 170 patients were aged 70 years or older.

Table 3: MRI diagnoses of 418 consecutive patients examined for non-trauma reasons.

\begin{tabular}{|l|l|}
\hline Diagnosis & No of patients \\
\hline Normal & 184 \\
\hline Bone lesions & 22 \\
\hline Bone cysts & 10 \\
\hline Paget's disease & 6 \\
\hline Enchondroma & 2 \\
\hline Fibrous dysplasia & 3 \\
\hline Transient osteoporosis & 1 \\
\hline Soft tissue lesions & 39 \\
\hline Abscess & 10 \\
\hline Bursitis & 14 \\
\hline Ganglion cysts & 3 \\
\hline Calcifications & 4 \\
\hline Lipoma & 2 \\
\hline Tendinopathy & 5 \\
\hline Neurofibromatosis & 1 \\
\hline Joint disease & 173 \\
\hline Coxarthrosis & 166 \\
\hline Sacroiliitis & 4 \\
\hline Symphysitis & 3 \\
\hline Total & 418 \\
\hline There were no & 418 \\
\hline
\end{tabular}

There were no pelvic fractures in 418 patients examined with MRI for non-trauma reasons.

special interest in hip and pelvic fracture diagnosis. The patients were scored for presence or absence of signals indicating fracture. The final diagnoses are MRI diagnoses.

Four 1.5T MRI scanners were used (Table 1). Coronal STIR and T1 sequences of both hips and the entire pelvis had been performed in all patients. The STIR sequences were TR 4000-4500 and TE 54-112 ms. The T1-weighted sequences were TR 505-600 and TE 14-20 ms. Slice thickness was 4-5 $\mathrm{mm}$.

\section{Results}

170 patients were aged 70 or older of which 51 were over 79 (Table 2). The reviewers agreed on that there were no MRI signals indicating pelvic fractures in any of the study subjects, thus no disagreement on fracture diagnosis. The final diagnoses were 184 normal, 173 patients with joint disease of which 166 had various degrees of coxarthrosis. There were benign bone lesions in 22 and benign soft tissue lesions in 39 patients (Table 3 ).

\section{Discussion}

Four 1.5T MRI scanners were used with somewhat varying acquisition data with $4-5 \mathrm{~mm}$ slices. There were no MRI signals indicating any skeletal lesion. Technically there is no reason to assume that fractures were overlooked. All patients in the current study were selected for MRI of the whole pelvis and hips and care was taken to include patients without any evidence of trauma and systemic diseases including osteoporosis. We have found no reports on pelvic fractures without foregoing minor or major trauma in elderly without systemic disease or osteoporosis. MRI is reported as very sensitive in detecting pelvic fractures $[1,3,4]$. Collin, et al. showed that $42 \%$ of 316 patients had exclusively pelvic fractures after trauma to the hip which emphasizes that pelvic fractures in elderly are associated with trauma [1]. As no fracture was detected age stratification did not add any value to the study except to demonstrate the size of the age groups. It is of interest to note that even in the 170 patients in the age interval $70-92$ years $(41 \%)$ where decreased quality of bone is not uncommon no fractures were found.

Several scanners were used but with fairly uniform protocol with similar acquisition data and both coronal STIR and T1-sequences. In conclusion, we are convinced that in a normal middle- or older-age population without systemic disease there are no pelvic fractures except after trauma.

\section{Compliance with Ethical Standards}

The study was approved by the Regional Board of Ethics.

\section{Conflict of Interest}

The authors declares that they have no conflict of interest.

\section{References}

1. Collin D, Geijer M, Göthlin JH (2016) Prevalence of exclusively and concomitant pelvic fractures at magnetic resonance imaging of suspect and occult hip fractures. Emerg Radiol 23: 17-21.

2. Kannus P, Palvanen M, Niemi S, Parkkari J, Järvinen M (2000) Epidemiology of osteoporotic pelvic fractures in elderly people in Finland: Sharp increase in 1970-1997 and alarming projections for the new millennium. Osteoporos Int 11: 443-448.

3. Cabarrus MC, Ambekar A, Lu Y, Link TM (2008) MRI and CT of insufficiency fractures of the pelvis and the proximal femur. AJR Am J Roentgenol 191: 995-1001.

4. Henes FO, Nüchtern JV, Groth M, Habermann CR, Regier M, et al. (2012) Comparison of diagnostic accuracy of Magnetic Resonance Imaging and Multidetector Computed Tomography in the detection of pelvic fractures. Eur $\mathrm{J}$ Radiol 81: 2337-2342. 